\title{
X-Ray Reflectivity Measurements of Surface Layering in Liquid Mercury
}

\author{
O. M. Magnussen and B. M. Ocko \\ Department of Physics, Brookhaven National Laboratory, Upton, New York 11973 \\ M. J. Regan, K. Penanen, and P. S. Pershan \\ Division of Applied Science and Department of Physics, Harvard University, Cambridge, Massachusetts 02138 \\ M. Deutsch \\ Department of Physics, Bar-Ilan University, Ramat-Gan 52100, Israel
}

(Received 9 January 1995)

\begin{abstract}
The surface normal structure of the mercury liquid-vapor interface has been investigated by measuring the x-ray reflectivity out to a momentum transfer of $q_{z}=2.5 \AA^{-1}$. The results provide direct experimental proof of surface layering in liquid metals. The layer spacing is given by the atomic dimensions of the $\mathrm{Hg}$ atoms. The minimum layer width agrees well with the predictions of capillary wave theory; the layering amplitude decays into the bulk with a characteristic length of $3-3.5 \AA$, which is close to the decay length of the bulk pair correlation function.
\end{abstract}

PACS numbers: 61.10.-i, 61.25.Mv, 68.10.-m

At the free surface of a liquid metal the density changes from a thin atomic vapor to a dense liquid over a distance of only a few atomic diameters [1]. This rapid vertical density variation is accompanied by a similarly rapid change in the effective interatomic interaction potential. The interface density profile is therefore ideally suited to test the extensive body of theoretical studies and simulations carried out over the last decades. Many of these studies predict layering at the surface to a depth of a few atomic diameters in several liquid metals [2-6], including mercury, in strong contrast to simple nonmetallic liquids where monotonic profiles have been observed $[7,8]$.

Considerable progress towards angstrom-resolution determination of the structure of liquid metal surfaces has been achieved in recent years using x-ray reflectivity and grazing incidence $\mathrm{x}$-ray diffraction techniques [9-13]. The reflectivity studies confirmed a relatively narrow interface region but were inconclusive with respect to surface layering due to their limited $q_{z}$ range. If layering indeed occurs, a Bragg-like peak should be observed at $q_{z} \approx 2 \pi / a$, where $a$ is of the order of the molecular spacing. For liquid metals, where $a \approx 3 \AA$, this entails measurements out to $\geq 2 \AA^{-1}$. The best previous measurements, performed by Bosio et al. [10] on $\mathrm{Hg}$ with a laboratory x-ray source, extended only to $q_{z}=0.75 \AA^{-1}$ and, hence, could not unambiguously resolve surface layering.

Here we report an x-ray reflectivity study of the surface of liquid mercury extending to $q_{z}=2.5 \AA^{-1}$. The measurements were carried out at a wavelength $\lambda=1.227 \AA$ using the Harvard-Brookhaven liquid surface diffractometer at beam line X22B of the National Synchrotron Light Source. The glass sample cell was composed of a lower compartment containing the sample through $(60 \mathrm{~mm} \times$ $20 \mathrm{~mm} \times 7 \mathrm{~mm}$ ) and an upper preparation compartment. After cleaning the cell with concentrated nitric acid and ultra pure water (Milli-Q), the sample compartment went through several cycles of hydrogen filling (zero grade purity) and evacuation. Subsequently, pure $\mathrm{Hg}$ ( $8 N$ bulk purity) was filled in the upper vessel, degassed for $30 \mathrm{~min}$, and finally dropped via a teflon needle valve into the glass trough up to a level several mm higher then the rim. Kept under hydrogen, the $\mathrm{Hg}$ surface is stable for more than $24 \mathrm{~h}$, as verified by the high reproducibility of the measured reflectivity curves.

To minimize vibrational surface excitations the cell was mounted on an active vibration isolation table, which in turn was mounted on the spectrometer. For measurements in the range $q_{z}<0.4 \AA^{-1}$ the incident beam was collimated to a vertical angular divergence of $0.07 \mathrm{mrad}$ and limited in height by a $0.05 \mathrm{~mm}$ slit $240 \mathrm{~mm}$ upstream of the sample to keep the footprint of the incident beam smaller than the sample size. For higher $q_{z}$ the slits were gradually opened to increase the reflected signal. The negligible increase in the divergence of the reflected beam, as compared to the incident beam, indicated a flat $\mathrm{Hg}$ surface and an efficient elimination of vibrational pickup from the environment by the isolation table.

To obtain the specular reflectivity, intensity measurements were carried out along the specular axis $\left(2 \theta=0^{\circ}\right)$ and by moving the detector by $2 \theta=0.6^{\circ}$ (i.e., several times the resolution width) out of the plane of reflection. Since the diffuse background depends only weakly on $2 \theta$, normalized by the incident beam intensity.

Figure 1(a) shows the absolute reflectivity $R$ of liquid mercury at room temperature (circles and plus signs) along with the theoretical reflectivity $R_{f}$ for a perfectly flat surface (solid line), calculated from the Fresnel law of optics. Also shown is a theoretical reflectivity for an interface broadened by the $\mathrm{Hg}$ atomic form factor and by thermally excited capillary waves (dotted line). The reflectivity falls from close to unity below the critical angle [14] to $3 \times 10^{-8}$ at $q_{z}=-2.3 \AA^{-1}$, yet it is always 


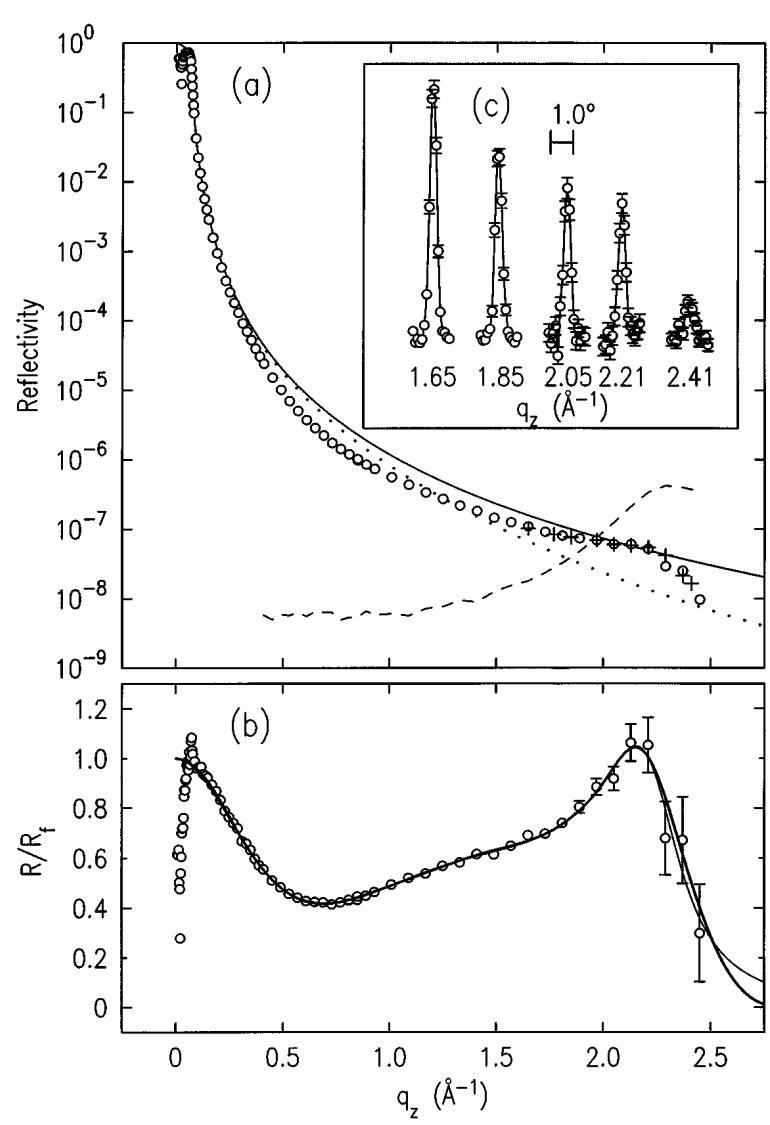

FIG. 1. (a) X-ray reflectivity of the free surface of liquid mercury at room temperature under $\mathrm{H}_{2}(\mathrm{O})$; (+) indicates values calculated from the transverse $2 \theta$ scans in (c). In addition, the Fresnel reflectivity $R_{f}$ for an ideally flat surface (-), the reflectivity of an interface with a roughness given by the convolution of the $\mathrm{Hg}$ atomic form factor and a Gaussian of $0.8 \AA$ width $(\ldots)$, and the background intensity (-- ) are shown. (b) Experimental data $(\bigcirc)$ and two fits $(-)$ of the ratio $R / R_{f}$. (c) Selected $2 \theta$ scans across the reflected beam at various $q_{z} \geq 1.5 \AA^{-1}(-0.8 \leq 2 \theta \leq 0.8$, background subtracted).

within a factor of 2 of $R_{f}$. This clearly indicates a surface roughness smaller than the atomic diameter.

Figure 1 (b) shows the ratio $R / R_{f}$ for our data (circles). This ratio decreases monotonically from unity all the way to $q_{z}=0.6 \AA^{-1}$, flattens out over a large range of $q_{z}$, and then exhibits a broad peak at $q_{z}=2.15 \AA^{-1}$. Above $2.5 \AA^{-1}$ the reflectivity drops below the sensitivity of our experiment $\left(\approx 10^{-8}\right)$. Capillary wave models alone are insufficient to explain these nonmonotonic features $[7,8]$. Futhermore, the broad peak at $q_{z}=2.15 \AA^{-1}$ strongly resembles similar features in reflectivity measurements of surface induced layering in liquid crystals [15] and hence suggests a layered mercury density profile at the interface. Finally, we note that the reflectivity data by Bosio et al. [10] measured at the $\mathrm{Hg}$ vapor interface below $0.75 \AA^{-1}$ is in good agreement with our data.

The short-range order within the bulk liquid gives rise to a peak in the liquid structure factor at $2.3 \AA^{-1}$ which strongly increases the contribution of diffuse scattering to the background intensity [dashed line in Fig. 1(a)]. In order to characterize and to eliminate this background, measurements at $q_{z}>1.5 \AA^{-1}$ were complemented by scans perpendicular to the reflection plane, i.e., $2 \theta$ scans [examples are shown in Fig. 1(c)]. These $2 \theta$ profiles exhibit identical, resolution limited line shapes. The integrated intensities of these beam profiles [shown in Fig. 1(a) as plus signs] are in excellent agreement with the background subtracted reflectivity curve (shown as circles). Thus, the peak in $R / R_{f}$ at $2.15 \AA^{-1}$ is a genuine product of the atomic structure at the liquid-vapor interface.

The ratio $R / R_{f}$ is directly related to the total electron density along the surface normal $\langle\rho(z)\rangle$ (averaged within the surface plane) through the equation

$$
R\left(q_{z}\right) / R_{f}\left(q_{z}\right) \approx\left|\left(1 / \rho_{\infty}\right) \int d z[\partial\langle\rho(z)\rangle / \partial z] e^{i q_{z} z}\right|^{2},
$$

where $\rho_{\infty}$ is the bulk electron density [16]. Since most of the electrons are located in the ion cores, $\langle\rho(z)\rangle$ is a good approximation to the ionic interface profile. The broad peak at $q_{z}=2.15 \AA^{-1}$ can only be fitted by an oscillatory component in $\langle\rho(z)\rangle$; its magnitude and width is related to the number and strength of these oscillations. These features cannot be produced by monotonic profiles or profiles exhibiting a single layer. A simple nonmonotonic parametrization based on an error function superimposed by an exponentially decaying sine wave, which has been successfully employed for liquid crystal data [15], reproduces the peak at $q_{z}=2.15 \AA^{-1}$ but fails to describe the data at low $q_{z}$. Hence more sophisticated models are necessary to obtain quantitative information on the density profile.

To insure model-independent results two different parametrizations of $\langle\rho(z)\rangle$ were used to fit the experimental data. The first employs a distorted semiinfinite crystal lattice (with lattice spacing $d$ ) where the root-mean-square (rms) displacement amplitude $\sigma=\sqrt{\sigma_{n}^{2}+\sigma_{0}^{2}}$ of the atoms from their lattice positions diverges with increasing distance $z$ from the surface. This gives rise to an oscillatory density profile close to the surface and a uniform density far below it. The reflectivity according to this model is given by

$$
\frac{R\left(q_{z}\right)}{R_{f}\left(q_{z}\right)} \approx\left|\frac{f\left(q_{z}\right)+f^{\prime}}{Z+f^{\prime}} d q_{z} e^{-\sigma_{0}^{2} q_{z}^{2} / 2} \sum_{n=1}^{\infty} \rho_{n} e^{i q_{z} p_{n}} e^{-\sigma_{n}^{2} q_{z}^{2} / 2}\right|^{2},
$$

where $f\left(q_{z}\right)$ and $f^{\prime}$ denote the $\mathrm{Hg}$ atomic form factor and the dispersion correction at $\lambda=1.227 \AA^{-1}$, respectively, and $Z_{\mathrm{Hg}}=80$. Choosing the rms displacement amplitude of the atoms in layer $n$ as $\sqrt{\sigma_{n}^{2}+\sigma_{0}^{2}}=\sqrt{n \bar{\sigma}^{2}+\sigma_{0}^{2}}$, where $\sigma_{0}$ and $\bar{\sigma}$ are constants, the semi-infinite sum can be solved analytically for uniformly spaced layers ( $p=$ $n d)$. Numerically, the corresponding density profile then approaches asymptotically an exponentially decaying sine wave for $z \rightarrow \infty$. Allowing for different layer spacings, different layer densities $\rho_{n}$, and different layer widths $\sigma_{n}$ only in the first $N$ layers, the sum in Eq. (2) reduces to 


$$
\sum_{n=1}^{\infty} \rho_{n} e^{i q_{z} p_{n}} e^{-\sigma_{n}^{2} q_{z}^{2} / 2}=\left[e^{\bar{\sigma}^{2} q_{z}^{2} / 2-i q_{z} d}-1\right]^{-1}+\sum_{n=1}^{N}\left[\rho_{n} e^{i q_{z} p_{n}} e^{-\sigma_{n}^{2} q_{z}^{2} / 2}-e^{-n \bar{\sigma}^{2} q_{z}^{2} / 2+i q_{z} d}\right] .
$$

Fits to the experimental data revealed that the first layer deviates from a simple Gaussian and exhibits a broader tail towards the vapor side of the interface. To model this asymmetry, the first layer was described by $2-3$ terms in the finite sum in Eq. (3), spaced at close distance. Corrections for other than the first layer did not improve the fit [i.e., $N \leq 3$ in Eq. (3)].

In the second model the interface profile is derived from the error-function density profile, which has been previously used to model simple liquid surfaces [7,8]. An error function of width $\sigma_{\text {erf }}$ is superimposed by distortions $\xi_{n}(z)$ periodically positioned at $p_{n}=n d$, i.e., $\langle\rho(z)\rangle / \rho_{\infty}=\operatorname{erf}\left(z / \sigma_{\text {erf }}\right)+\sum_{n=1}^{N} \xi_{n}(z)$ with

$$
\begin{aligned}
\xi_{n}(z)=\frac{a_{n}}{\sqrt{2 \pi} \sigma_{n}}[ & \rho_{n} e^{-\left(z-p_{n}\right)^{2} / 2 \sigma_{n}^{2}}-\frac{1}{2} e^{-\left(z-p_{n}+\Delta p_{n}\right)^{2} / 2 \sigma_{n}^{2}} \\
& \left.-\frac{1}{2} e^{-\left(z-p-\Delta p_{n}\right)^{2} / 2 \sigma_{n}^{2}}\right]
\end{aligned}
$$

and $\Delta p_{n}=\frac{1}{2}\left[p_{n-1}-p_{n+1}\right] / 2$. This choice of $\xi_{n}(z)$ conserves mass since $\int_{-\infty}^{\infty} \xi_{n}(z) d z=0$ for $\rho_{n}=1$. The widths are chosen as $\sigma_{n}=\Delta p_{n} / 2$. For the inner layers $(3 \leq n \leq N)$ the layer spacing was kept constant $\left(p_{n}=n d\right)$ and the layering amplitude $a_{n}$ set to decay exponentially with $z$. For the first two or three layers deviations were allowed in $a_{n}$ from the exponential form, in $\rho_{n}$ from unity, and in the layer spacings from $d$. Allowing such deviations for $n>3$ did not improve the fits.

Despite the vastly different origins of these two models, both models give excellent, almost indistinguishable fits of the experimental $R / R_{f}$ data and very similar density profiles, as shown in Fig. 1(b). The corresponding electron density profiles $\langle\rho(z)\rangle$ are shown in Fig. 2(a). Although the limited $q_{z}$ range poses some uncertainties on the numerical values of the parameters, all good fits share the following properties: $\langle\rho(z)\rangle$ exhibits significant layering with a spacing of $3.05 \pm 0.15 \AA$ between the first and the second layer and $2.76 \pm 0.20 \AA$ spacing between all subsequent layers. The relative density of each layer (integrated over the layer thickness) matches the bulk density within the experimental error $\left(\rho_{1}=1.09 \pm 0.12\right.$, $\rho_{n}=1.00 \pm 0.04$ for $n \geq 2$ ); the widths of all but the first layer can be described by $\sigma_{n}=\left[\sigma_{0}^{2}+n \bar{\sigma}^{2}\right]^{1 / 2}$ (first model) with $\sigma_{0}=0.95 \pm 0.05 \AA$ and $\bar{\sigma}=0.50 \pm 0.05 \AA$. As illustrated in Fig. 2(b) (triangles), the layering amplitudes $\left[\rho\left(z_{\text {peak }}\right)-\rho_{\infty}\right] / \rho_{\infty}$ can be described by an exponential decay into the bulk with a characteristic length of 3-3.5 A. The first layer is asymmetric with a broader tail towards the interface (see above). The largest (modelindependent) uncertainty in the results is the contribution of this broad component ( $\sigma \approx 2-4 \AA$ ) to the first layer. As demonstrated by the two fits shown in Figs. 1(b) and 2(a), the resulting $R / R_{f}$ are almost identical, even if the amplitudes and the widths of the broad components are quite different. Minor deviations are seen only at $q_{z}>2.2 \AA^{-1}$ where the experimental error is large.

These results can be rationalized well within our current knowledge of liquid-metal surfaces. The intrinsic layering profile is broadened by thermally excited capillary waves. Capillary wave theory predicts a broadening of 0.8 $1.0 \AA$ in our case given the surface tension and molecular size of $\mathrm{Hg}(\gamma=485 \mathrm{mN} / \mathrm{m} ; a=3 \AA)$, temperature and experimental resolution $(6.6 \mathrm{mrad}$ angular detector acceptance) [8]. This agrees well with the 1.1-1.4 widths obtained from the first model for the first four layers. Hence, the width of the surface layers on $\mathrm{Hg}$ can be attributed largely to thermal excitation of capillary waves at the interface. With the exception of the first, the interlayer spacings are smaller than the nearest neighbor spacing at the bulk liquid but larger than the layer spacing in a crystalline close-packed arrangement. This is expected since the large thermal amplitude should inhibit a well-ordered atomic arrangement, whereas a partial penetration of the atoms into the neighboring layers is possible. The enlarged first interlayer spacing may be caused by the strong anharmonicity in the surface-normal component of the interatomic potential at the surface.

Monte Carlo (MC) simulations of $\mathrm{Hg}$ clusters [2,3] predict about four layers with a uniform spacing of $2.71 \AA$,
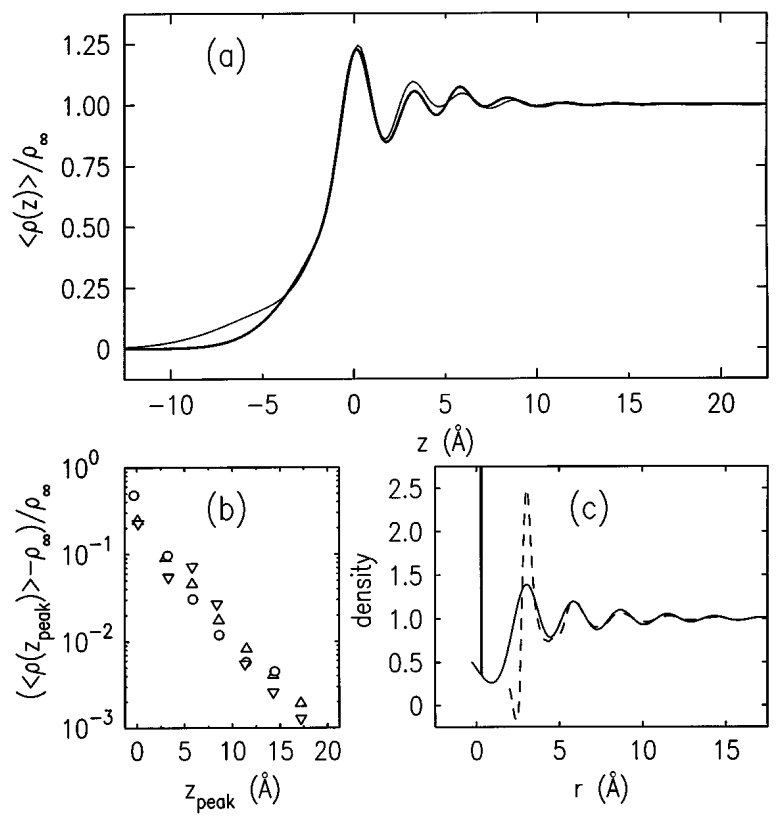

FIG. 2. (a) Normalized electron density profiles $\langle\rho(z)\rangle$ corresponding to the two fits in Fig. 1(b). (b) Relative layering amplitudes and peak positions for the density profiles shown in bold $(\nabla)$ and thin $(\triangle)$ lines in (a). Also shown are the same quantities for the bulk correlation function $g(r)$ convoluted by a $0.8 \AA$ wide Gaussian $(\bigcirc)$. (c) Intrinsic, deconvoluted surface density profile (-) and the bulk correlation function $g(r)(---)$; for discussion see text. 
in agreement with the experimental results. They also show a low-density layer of vaporized $\mathrm{Hg}$ atoms on top of the first liquid layer. This could account for the broad tail of the interface profile discussed above; other explanations, however, such as the anharmonic interaction potential for ions in the first layer or a low-density adlayer of hydrocarbons cannot be excluded. Contrary to our data, the simulations produce extremely well ordered $\mathrm{Hg}$ layers with layering amplitudes up to three times the $\mathrm{Hg}$ bulk density and layer widths up to $0.3 \AA$. This discrepancy may be due to an inaccurate pseudopotential or the finite size of the $\mathrm{Hg}$ cluster used in the simulations. Considerably smaller amplitudes were found in recent MC simulations of $\mathrm{Na}$ [5] and second-order perturbation theory calculations for alkali metals $[4,6]$ and $\mathrm{Al}[6]$.

The MC simulations [3,5] show that due to its strong dependence on the electron density the effective interaction energy acts as a hard wall at the interface. Layering arises from packing the atoms against this wall. Neglecting the interface broadening by thermal capillary waves (i.e., assuming the first layer as perfectly sharp and at $z=0$ ) this packing may be compared to the packing around a $\mathrm{Hg}$ atom in the bulk, which is described by the pair correlation function $g(r)$. However, note that (a) $g(r)$ is averaged over a spherical shell, $\langle\rho(z)\rangle$ over the surface plane; (b) $g(r)$ reflects an isotropic interatomic potential, while for $\langle\rho(z)\rangle$ the $z$ component of the interatomic potential is highly anisotropic close to the surface; and (c) the first peak in $g(r)$ is related to the well-defined hardcore diameter of the ions, whereas in $\langle\rho(z)\rangle$ atoms in all layers, including the first, can penetrate into neighboring layers. These differences are pronounced near the origin but become less important for larger $r$ and $z$, respectively. Hence, the agreement for $g(r)$ and $\langle\rho(z)\rangle$ should be best in the asymptotic limit. To obtain the intrinsic layering, $\langle\rho(z)\rangle$ was deconvoluted by the $\mathrm{Hg}$ form factor and a Gaussian of width $0.8 \AA$ representing the capillary wave broadening. In Fig. 2(c) the deconvoluted $\langle\rho(z)\rangle$ and $g(r)$ measured by Waseda [17] are plotted on the same scale. To verify the asymptotic agreement we aligned the peaks at $\approx 3 \AA$, rather than the $\delta$ functions at $g(0)$ and near the origin of $\langle\rho(z)\rangle$. In addition, the peak positions and amplitudes, which result if $g(r)$ is convoluted with a Gaussian of width $0.8 \AA$, are shown in Fig. 2(b) (circles). With the exception of the first peak, the two curves in Fig. 2(c) are in good agreement. The positions of the second and all following peaks in $g(r)$ match the positions of the layers in $\langle\rho(z)\rangle$ within the experimental error. Furthermore, the decay lengths and, within a factor of 2 , the amplitude agree. This suggests that the short-range order in bulk liquid metals and the layering at the surface are closely related and, in turn, indicates that the latter is primarily a simple geometric consequence of the physical requirement to form a sharp interface.

In summary, our results provide the only conclusive experimental proof of surface layering in liquid metals to date. The widths of the layers are in good agreement with capillary wave theory; the interlayer spacings and the decay length of the layering amplitudes resemble the local ordering in the bulk liquid described by the pair correlation function. Surface layering may not be restricted to mercury but probably is a more general phenomenon in liquid metals. This is indicated by very recent observations of similar $\mathrm{x}$-ray reflectivity features in liquid gallium [18].

The authors thank B. W. Lafler of the BNL glass shop for help in the design and construction of the cell and D. Turnbull for helpful discussions. We acknowledge support by the Divisions of Material Sciences, U.S. Department of Energy and by the National Science Foundation under Contracts No. DE-AC02-76CH00016, No. DE-FG02-88-ER45379, No. NSF-DMR-91-13782, and No. NSF-DMR-89-20490.

[1] N. H. March, Liquid Metals (Cambridge University Press, New York, 1990), p. 23.

[2] M.P. D'Evelyn and S. A. Rice, J. Chem. Phys. 78, 5081 (1983).

[3] S. A. Rice, Proc. Natl. Acad. Sci. U.S.A. 84, 4709 (1987).

[4] M. Hasegawa, J. Phys. F 18, 1449 (1988).

[5] A. Ishida, M. Hasegawa, and M. Watabe, J. Non-Cryst. Solids 117/118, 650 (1990).

[6] J. Gómez and E. Chacón, Phys. Rev. B 46, 723 (1992); 49, 11405 (1994).

[7] A. Braslau, P. S. Pershan, G. Swislow, B. M. Ocko, and J. Als-Nielsen, Phys. Rev. A 38, 2457 (1988).

[8] B. M. Ocko, X.Z. Wu, E. B. Sirota, S. K. Sinha, and M. Deutsch, Phys. Rev. Lett. 72, 242 (1994).

[9] B. C. Lu and S. A. Rice, J. Chem. Phys. 68, 5558 (1978).

[10] L. Bosio, R. Cortès, A. Defrain, and M. Oumedzine, J. Non-Cryst. Solids 61/62, 697 (1984); L. Bosio, R. Cortès, G. Folcher, and M. Froment, J. Electrochem. Soc. 139, 2110 (1992).

[11] S. W. Barton, B. N. Thomas, F. Novak, P. M. Weber, J. Harris, P. Dolmer, J. M. Bloch, and S. A. Rice, Nature (London) 321, 685 (1986); B. N. Thomas, S. W. Barton, F. Novak, and S. A. Rice, J. Chem. Phys. 86, 1036 (1987).

[12] E. H. Kawamoto, S. Lee, P.S. Pershan, M. Deutsch, N. Maskil, and B. M. Ocko, Phys. Rev. B 47, 6847 (1993).

[13] E. B. Flom, M. Li, A. Acero, N. Maskil, and S. A. Rice, Science 260, 332 (1993).

[14] At very small incident angles $\left(q_{z}<0.05 \AA^{-1}\right)$ the large footprint causes spillover of the incident beam over the sample edge and consequently deviations from $R_{f}$. Hence, these data were not used in the fitting.

[15] P. S. Pershan, A. Braslau, A. H. Weiss, and J. Als-Nielsen, Phys. Rev. A 35, 4800 (1987).

[16] P. S. Pershan and J. Als-Nielsen, Phys. Rev. Lett. 52, 759 (1984).

[17] Y. Waseda, The Structure of Non-Crystalline Materials (McGraw-Hill, New York, 1980), p. 23.

[18] M. J. Regan, E. H. Kawamoto, S. Lee, P.S. Pershan, N. Maskil, M. Deutsch, O. M. Magnussen, and B. M. Ocko (to be published). 